\title{
VALORIZATION OF AGRICULTURAL WASTES IN MOROCCO: EXTRACTION OF CELLULOSE NANOCRYSTALS FROM SUGARCANE BAGASSE FIBERS AS CASE STUDY
}

\section{NASSIMA EL MIRI ${ }^{1}$, MOUNIR EL ACHABY ${ }^{2}$ and ABDERRAHIM SOLHY ${ }^{2}$}

${ }^{1}$ Faculté des Sciences et Techniques

Université Hassan II-Casablanca

Casablanca 20650

Morocco

${ }^{2}$ Center for Advanced Materials (CAM)

Université Mohammed VI Polytechnique (UM6P)

Lot 660-Hay Moulay Rachid

43150 Ben Guerir

Morocco

e-mail: mounir.elachaby@um6p.ma

abderrahim.solhy@um6p.ma

\begin{abstract}
Sugar production is a main agro-based industry in Morocco that generates various solid wastes. The sugarcane bagasse is one of the important wastes of this industry, which is mainly used in combustion for energy production within the sugar production plant. It is very interesting to valorize this waste for the production of high value added nanomaterials. The aim of this work is to isolate of cellulose nanocrystals (CNC) from Moroccan sugarcane bagasse (SCB) by chemical methods. The pure cellulose was produced via alkaline and bleaching
\end{abstract}

Keywords and phrases: agro-industrial residue, sugarcane bagasse, cellulose, cellulose nanocrystals, acid hydrolysis.

Received November 25, 2015

(C) 2015 Scientific Advances Publishers 
treatments, hence suitable for extracting of CNC. Acid hydrolysis process has been used to isolate the CNC using sulphuric acid at a concentration of $9 \mathrm{M}$ for $30 \mathrm{~min}$ and hydrochloric acid at two concentrations of $2 \mathrm{M}$ and $3 \mathrm{M}$ for $120 \mathrm{~min}$. Several techniques, including Fourier transformed infrared, X-ray diffraction, thermogravimetric analysis, scanning electron microscopy, and atomic force microscopy; were used to characterize the products at different stages of treatment. The effect of acid type, hydrolysis time and acid concentration on the morphology, dimensions, crystallinity, and thermal stability of CNC has been evaluated and discussed through this work. The obtained results show that the as-extracted CNC exhibit needle-like nanoparticles with a diameter range of 3 $25 \mathrm{~nm}$ and a length of $100-400 \mathrm{~nm}$ depending on the hydrolysis conditions. This work is achieved in order to valorize the sugarcane bagasse that growth in Morocco in large quantity, which is mainly used actually in combustion for electricity production. In overall, the CNC obtained from Moroccan sugarcane bagasse have excellent properties and can be potentially used in various applications.

\section{Introduction}

The vegetal biomass has many applications since it can be used to produce foods and generate electricity as well liquid biofuels for our transportation needs. The biomass can also provide a wide access to new and promising materials and molecular platform with highly added value [1]. For a sustainable use of biomass, the valorization of biomass-derived waste for various applications is became an urgent need. Unfortunately, the abundant Moroccan biomass, such as bagasse, straw, wood, sawdust, nut shells, olive husks, palm kernel cake, etc. is not developed yet and its valorization today is limited in the generation of electricity by combustion or it use as feed for livestock.

The sugar industry generates two main by-products: molasses and bagasse. The latter is a fibrous by-product obtained after crushing of sugarcane to extract its juice and its production varies from one country to another and associated with the quantity of sugarcane produced. This by-product is produced annually in large quantities every year throughout the world, Brazil [2, 3], India [4], Cuba [2], China [2, 5], México [3], Indonesia [6] and Colombia [7] and Morocco. The particularity of this Moroccan renewable source is the climate and the weather 
conditions which can be considered two major global ecological concerns with a great potential to alter the shape and structure of sugarcane bagasse and biomasses in general.

The SCB contains about 40-50\% of cellulose in its composition [8]. This characteristic suggests the possibility of using the SCB as biosourced materials to obtain the pure cellulose or cellulose nanocrystals (CNC) [9-14]. The extracted CNC which can be sphere-like, rod-like, ribbon-like, or needle-like shape, have lengths ranging from $100 \mathrm{~nm}$ up to $1-2 \mu \mathrm{m}$, and a diameter of about $5-25 \mathrm{~nm}[15,16]$.

The CNC can be separated by acid hydrolysis of pure cellulosic fibers or micro fibrillated cellulose $[11,17,18]$. The most common acid used is the sulphuric acid [13, 16-20], which was used for the first time by Nickerson and Habrle [21] more than half a century ago by the treatment of cotton for $30 \mathrm{~min}$ with boiling $2.5 \mathrm{~N}$ sulphuric acid. However, it significantly decreases the thermal stability of CNC. Since typical processing temperatures for thermoplastics rise above $200^{\circ} \mathrm{C}$, the thermal stability of these crystals is a key factor in order for them to be used as effective reinforcing materials [22]. In this case, the thermal stability can be recovered by partly neutralizing the sulphuric acid groups with strong bases such as sodium hydroxide [23]. Others acids were used such hydrobromic acid [24], phosphoric acid [22], and hydrochloric acid [22, 25, 26].

Recently, Mandal and Chakrabarty [13] reported the extraction of nanocellulose from SCB by using $60 \%(\mathrm{w} / \mathrm{v})$ sulphuric acid hydrolysis at $50^{\circ} \mathrm{C}$ for $5 \mathrm{~h}$. From their results, the nanocellulose in form of particles and rods has been obtained with nanometric size ranging from 35 to $90 \mathrm{~nm}$ in diameter. $\mathrm{Li}$ et al. [10] reported the isolation of nanocellulose from SCB by using high pressure homogenization in a homogeneous media, and the obtained nanocellulose had a circular shaped nanoparticules with a diameter in the range of $10-20 \mathrm{~nm}$. Bras et al. [14] reported the isolation of cellulose whiskers from bleached SCB kraft pulp by 65 wt\% sulphuric acid hydrolysis at $45^{\circ} \mathrm{C}$ for $45 \mathrm{~min}$. Their results revealed that the obtained whiskers have a form of rod-like nanoparticules with a length in the range $84-102 \mathrm{~nm}$ and a diameter in the range $4-12 \mathrm{~nm}$. Teixeira et al. 
[11] reported the effect of extraction time on the properties of CNC obtained from SCB by $6 \mathrm{M}$ sulphuric acid hydrolysis for 30 and $75 \mathrm{~min}$. Their results showed that the extracted CNC had needle-like structures with an average length of $255 \pm 55 \mathrm{~nm}$ and diameter of $4 \pm 2 \mathrm{~nm}$. They have also demonstrated that the increasing of extraction time from 30 to $75 \mathrm{~min}$ lead to a decrease in the thermal stability and cause some damage on the crystal structure. Consequently, geometrical and structural characteristics of CNC depend on the hydrolysis conditions such as type and concentration of acid, temperature and time as well as the original source of cellulose fibers [27].

The purpose of our study is to valorize the sugarcane bagasse that growth in Morocco country for the production of CNC. In this way, three main steps are involved, including alkaline and bleaching treatments and acid hydrolysis process. Acid hydrolysis has been carried out under different conditions by using sulphuric acid $\left(\mathrm{H}_{2} \mathrm{SO}_{4}\right)$ at a concentration of $9 \mathrm{M}$ for $30 \mathrm{~min}$ and hydrochloric acid $(\mathrm{HCl})$ at two concentrations of $2 \mathrm{M}$ and $3 \mathrm{M}$ for $120 \mathrm{~min}$. The effect of acid type, hydrolysis time and acid concentration on the morphology, dimensions, crystallinity, and thermal stability of CNC has been evaluated and discussed in this report.

\section{Materials and Experimental Details}

\subsection{Materials}

The SCB was provided by SUNABEL-COSUMAR Group, company located in the Gharb-Loukkos region of Morocco. Firstly, the SCB was ground using a precision grinder (Fritsch-Pulverisette 19, GmbH) equipped with a $250 \mu \mathrm{m}$ sieve. Then, this fraction was sifted in a $150 \mu \mathrm{m}$ sieve size (Fritsch mesh) to remove the small particles. The moisture content of the SCB was about $7 \mathrm{wt} \%$. Analytical grade chemicals used for extraction, bleaching, and hydrolysis were purchased from Sigma-Aldrich and used without further purification. 


\subsection{Treatment of SCB}

Firstly, a quantity of $15 \mathrm{~g}$ of grinded raw SCB and $300 \mathrm{ml}$ of deionized water were placed in Erlenmeyer flask for $1 \mathrm{~h}$ at $60^{\circ} \mathrm{C}$ under stirring. The obtained mixture was filtrated and washed several times with deionized water. Then, the obtained washed SCB was subjected to an alkaline treatment with a $4 \mathrm{wt} \% \mathrm{NaOH}$ solution at $80^{\circ} \mathrm{C}$ for $2 \mathrm{~h}$ under mechanical stirring. This treatment was done three times and after each treatment the fibers were filtered and washed with distilled water until the alkali was completely eliminated. A subsequent bleaching treatment was carried out to bleach the fibers. The solution used in this treatment consisted of equal parts of acetate buffer, aqueous chlorite (1.7 wt\% in water), and distilled water. The bleaching treatment was performed at $80^{\circ} \mathrm{C}$ for $4 \mathrm{~h}$ under mechanical stirring and was repeated four times. After each treatment, the fibers were filtered and washed with distilled water.

\subsection{Isolation of CNC}

Acid hydrolysis process has been used to isolate the CNC by using $\mathrm{HCl}$ (H-CNC) and $\mathrm{H}_{2} \mathrm{SO}_{4}$ (S-CNC) acids. In brief, for $\mathrm{HCl}$ hydrolysis, the bleached cellulose fibers were hydrolyzed by treatment with $2 \mathrm{M}$ $\left(\mathrm{H}-\mathrm{CNC}_{2 \mathrm{M}}\right)$ and $3 \mathrm{M}\left(\mathrm{H}-\mathrm{CNC}_{3 \mathrm{M}}\right) \mathrm{HCl}$ solutions at $80^{\circ} \mathrm{C}$ for $120 \mathrm{~min}$ under continuous stirring. After this time, the mixture was cooled to room temperature and the insoluble residue (CNC) was purified and washed until neutrality with repeated centrifugations (30 min, 12000rpm). After the centrifugation, the suspension was sonicated for $30 \mathrm{~min}$ for homogenization. For $\mathrm{H}_{2} \mathrm{SO}_{4}$ hydrolysis, $\mathrm{S}-\mathrm{CNC}$ were extracted following the method described by Habibi et al. [28]. Acid hydrolysis with 9M $(65 \%$, w/w $) \mathrm{H}_{2} \mathrm{SO}_{4}$ solution was applied to homogeneous suspension of bleached cellulose. The hydrolysis was carried out at $55^{\circ} \mathrm{C}$ for $30 \mathrm{~min}$ under continuous stirring. The suspension was diluted with ice cubs to stop the reaction and washed until neutrality by successive centrifugations (30 min, 12000rpm) at $15^{\circ} \mathrm{C}$ and dialyzed against distilled 
water. Afterward the S-CNC suspension was homogenized by sonication for $5 \mathrm{~min}$ in an ice bath to avoid overheating, which can cause desulfation of the sulphate groups on the surface of S-CNC.

\subsection{Characterization techniques}

Scanning electron microscopy (SEM) observations were recorded by using Quanta 200-ESEM, FEI instrument operating at $25 \mathrm{kV}$. Fourier transform infrared spectroscopy (FTIR) was performed on an ABB Bomem FTLA 2000 spectrometer equipped with a Golden Gate single reflection ATR accessory. The spectra were obtained with an accumulation of 16 scans in the range of $4000-400 \mathrm{~cm}^{-1}$ and with a resolution of $4 \mathrm{~cm}^{-1}$. X-ray diffraction (XRD) measurements of the samples were carried out by using a Bruker D8 Discover with the $\mathrm{Cu} \mathrm{K} \alpha$ radiation $(\lambda=1.54184 \mathrm{~nm})$. The crystallinity index $(\mathrm{CrI})$ of the material was determined by the Segal method [29, 18], with the equation as follows: $\operatorname{Cr} I=\left[\left(I_{002}-I_{\text {amorph }}\right) / I_{002}\right] \times 100$, where $I_{002}$ and $I_{\text {amorph }}$ are the peak intensities of crystalline and amorphous cellulose, respectively. The zeta potential measurement was carried out by using a Malvern Zetasizer Nano ZS instrument. Atomic force microscopy (AFM) measurement was carried out by using a Veeco Dimension ICON. Samples for AFM images were prepared by depositing a suspension of CNC onto a freshly cleaved mica surface and allowing them to dry in air. Thermogravimetric analysis (TGA) was performed on a TA Instrument Q500 Analyzer. A total of $15 \mathrm{mg}$ was settled in a platinum pan and heated under air from room temperature to $600^{\circ} \mathrm{C}$ at a rate of $10^{\circ} \mathrm{C} / \mathrm{min}$.

\section{Results and Discussion}

\subsection{Physical aspect and morphological analysis of purified SCB}

The pure cellulose, or bleached cellulose, has been obtained following two steps. Beginning with the first step which was the alkali treatment of raw SCB fibers, the main focus of this stage was to eliminate the whole 
non-cellulosic components (lignin, hemicelluloses, and pectin) [30]. During the alkaline treatment process, some alkali-labile linkages (ether and ester linkages) between lignin monomers or between lignin and polysaccharides may be broken. And this fact can be proven from the FTIR analysis of alkali-treated fibers [31]. The second step was the bleaching treatment which the main aim was to purify the cellulosic fibers obtained after the alkaline treatment from any residual lignin or other impurities. The resulting fibers obtained after the bleaching process might be contain pure cellulose.

Figure 1 shows the physical aspect of the raw SCB at different stages of the chemical treatment. Visually, the initial SCB fibers have a yellowbrown colour. However, yellow coloured ATB fibers were obtained after alkaline treatment, indicating that a great part of the initial noncellulosic components were removed by alkaline process. This treatment yielded about $45 \%$, which was calculated by the weight of the obtained ATB fibers divided by the initial weight of SCB fibers. After the bleaching treatment, the obtained BC fibers showed a clearly white colour, confirming that the residual non-cellulosic elements are totally removed under bleaching treatment. The yield of this bleached product was about $33 \%$ in regard to the initial amount of SCB. 

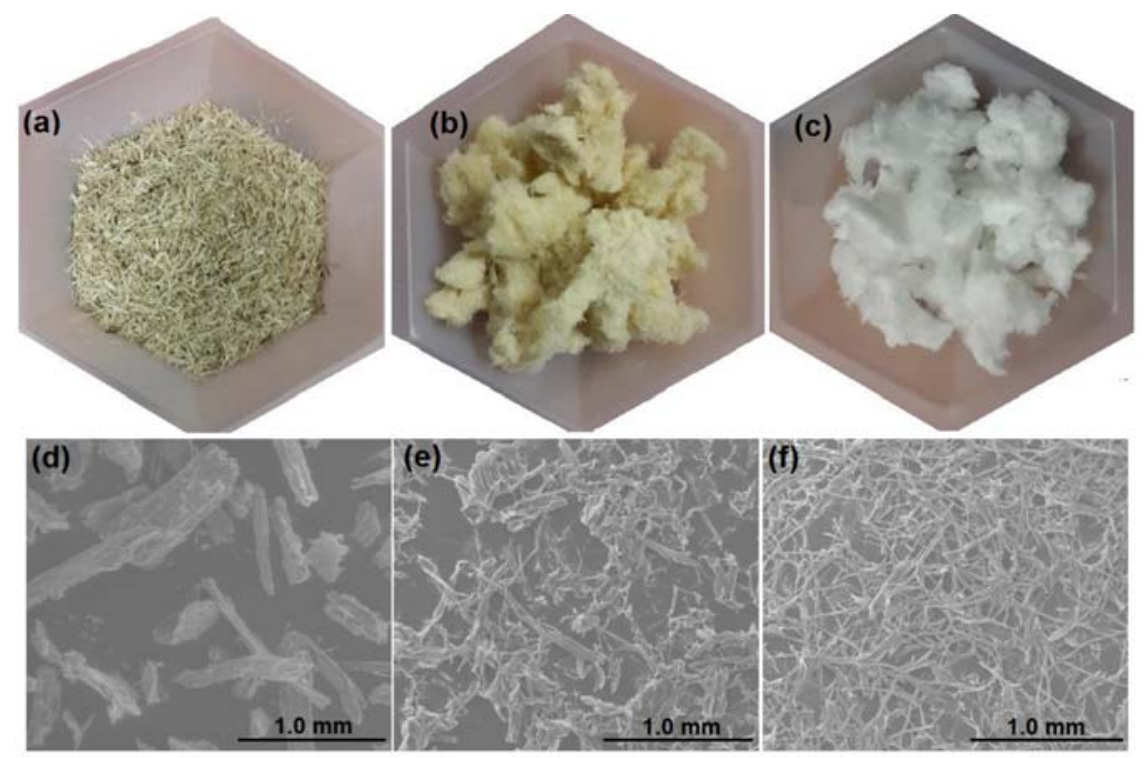

Figure 1. Photographs of (a) sugarcane bagasse (SCB), (b) alkali-treated bagasse (ATB), and (c) bleached cellulose (BC) samples. SEM micrographs of (d) SCB, (e) ATB, and (f) BC samples.

Raw SCB, ATB, and BC samples were analyzed by SEM in order to assess structural and morphological changes during the alkali and bleaching treatments. The obtained micrographs are shown in Figure 1. The micrographs revealed a significant changes in the fibrous structure under different chemical treatments. Indeed, the diameter of the raw grinded SCB fibers was much bigger and each fiber appears to be composed of several fibrils, which are associated in small bundles and cemented by non-cellulosic compounds, showing a complete and compact structure along each elementary SCB fiber; exhibiting an alignment in the fiber axis direction (Figure 1(d)). On subsequent treatment with alkali, the lignin is complexed and depolymerized, helping in defibrillation of fibers into individual small fibers, forming a highly entangled soft-flat shape and resulting in micrographs shown in Figure 1(e). After bleaching treatment, individual microfibrils are obtained with a smaller diameter $(15-20 \mu \mathrm{m})$ in comparison with raw SCB and ATB 
fibers. This is attributed to the total removal of lignin and hemicelluloses, resulting in dispersed and individualized microfibrils of pure cellulose (Figure 1(f)). Consequently, the individualization of these bleached cellulose microfibrils can favor its chemical reactions such as acidic hydrolysis [32].

\subsection{FTIR spectroscopy analysis of purified SCB and CNC}

The FTIR spectroscopy has been extensively used to study the changes that occur in the chemical composition during different stage of treatment, starting from raw SCB until the production of the $\mathrm{CNC}[8,13$, $30,33]$. The FTIR spectra of $\mathrm{SCB}, \mathrm{ATB}, \mathrm{BC}, \mathrm{H}-\mathrm{CNC}_{2 \mathrm{M}}, \mathrm{H}-\mathrm{CNC}_{3 \mathrm{M}}$, and S-CNC samples are represented in Figure 2.

Breaking down the SCB spectrum into individual peaks, the peak at $1742 \mathrm{~cm}^{-1}$ is attributed to the acetyl and uronic ester groups of hemicelluloses or the ester linkage of carboxylic group of ferulic and $p$-coumaric acids of lignin and/or hemicelluloses $[8,33]$, the peak at $1596 \mathrm{~cm}^{-1}$ can be assigned to the $\mathrm{C}=\mathrm{C}$ stretching vibrations from the aromatic ring of lignin $[8,17]$ and the peak at $1242 \mathrm{~cm}^{-1}$ is assigned to the $\mathrm{C}-\mathrm{O}$ out of plane stretching vibration of the aryl group in the lignin molecules. For the ATB fibers, the observed spectrum is rather similar to that of SCB, except the characteristic bands of hemicelluloses and lignin are reduced. This reveals that the complete removal of lignin and hemicelluloses components does not take place. However, after the bleaching step, we can observed that the characteristic bands of hemicelluloses and lignin molecules disappeared in the BC spectrum, confirming that the residual parts of these molecules were totally removed by bleaching treatment, and as result, the content of cellulose can be increased $[8,33]$. It must be also noted that there is a new band

appearing at $1099 \mathrm{~cm}^{-1}$ for $\mathrm{BC}$ spectrum, which is related to the $\mathrm{C}-\mathrm{O}$ at C-6 stretching [34]. 


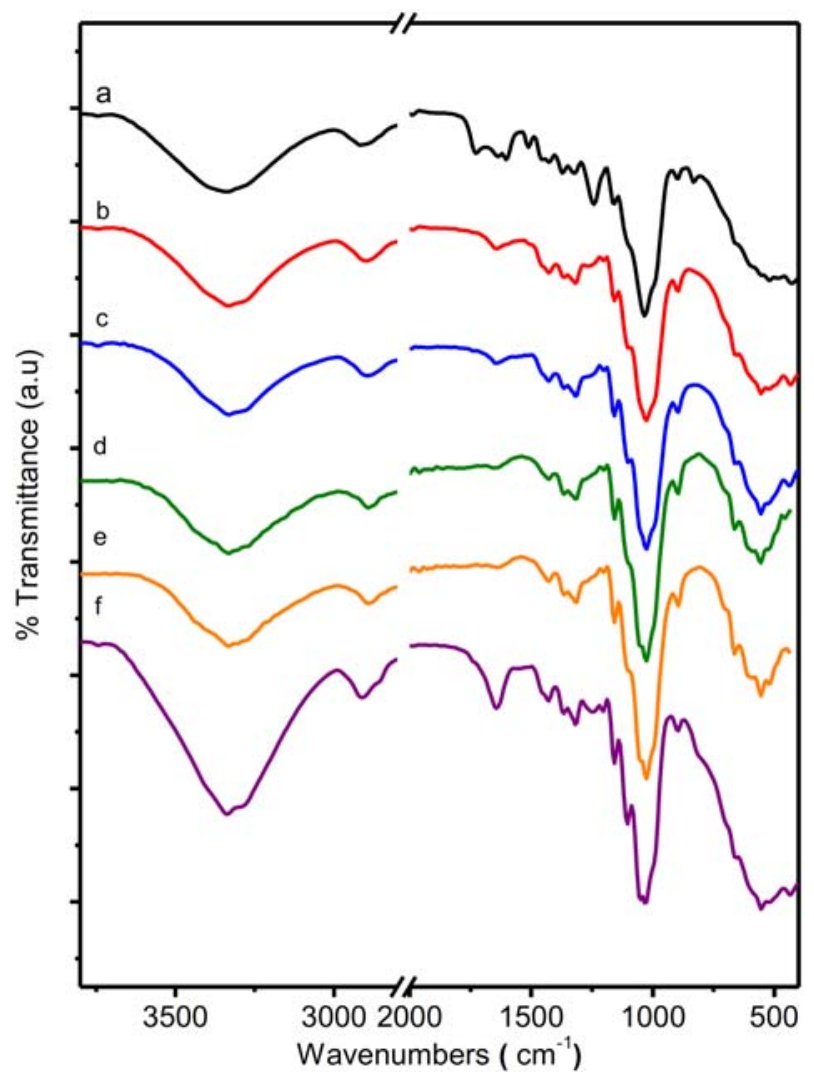

Figure 2. FTIR spectra of (a) SCB, (b) ATB, (c) BC, (d) $\mathrm{H}-\mathrm{CNC}_{2 \mathrm{M}}$, (e) $\mathrm{H}-\mathrm{CNC}_{3 \mathrm{M}}$, and $\mathrm{SCNC}$ samples.

Concerning the spectra of both $\mathrm{CNC}$ (H-CNC and S-CNC), it can be seen that there is no difference comparing with the spectrum of $\mathrm{BC}$ fibers, because no difference between $\mathrm{CNC}$ and $\mathrm{BC}$ in term of chemical compositions. In the other words, the major key of obtaining $\mathrm{CNC}$ is the bleaching step that leads to obtain pure cellulose (higher content of cellulose as possible) which makes the acid attack on the amorphous regions easier. Besides, although varying the acid type, the main differences in the FTIR spectra of S-CNC and $\mathrm{H}-\mathrm{CNC}$ are the intense peak of the adsorbed water in the S-CNC at $1639 \mathrm{~cm}^{-1}$ and the $1202 \mathrm{~cm}^{-1} \mathrm{~S}=\mathrm{O}$ sulphate peak, which indicates that hydrolyzing 
cellulose with sulphuric acid involves esterification of the hydroxyl groups during acid hydrolysis [35]. The surface charge density of the SCNC was determined by conductometric titration with sodium hydroxide [36], and the obtained average value is $162 \mathrm{mmol} / \mathrm{Kg}$, confirming the attachment of anionic sulphate groups into the surface of S-CNC after sulphuric acid treatment [33]. In contrast, for H-CNC obtained by the hydrochloric acid, the surface groups were hydroxyl groups.

In addition, the broad band of stretching vibrations of $\mathrm{CH}$ and $\mathrm{OH}$ groups within $3700-3000 \mathrm{~cm}^{-1}$ region was observed in all spectra, showing the principal functional groups found in lignocellulosic materials [34]. Moreover, the spectra of all samples showed the characteristic $\mathrm{C}-\mathrm{H}$ stretching vibration around $2893 \mathrm{~cm}^{-1}$. In addition, the band detected at $1649 \mathrm{~cm}^{-1}$ is due to the bending mode of the absorbed water $[8,33,34]$. The absorbed band at $1151 \mathrm{~cm}^{-1}$, which appears in all FTIR spectra, could be attributed to the $\mathrm{C}-\mathrm{C}$ ring breathing mode. Besides, the peaks at 1023 and $902 \mathrm{~cm}^{-1}$ are characteristics of the cellulose, and correspond to the $\mathrm{C}-\mathrm{O}-\mathrm{C}$ pyranose ring skeletal vibration and the glycosidic $\mathrm{C}_{1}-\mathrm{H}$ deformation with ring vibration contribution and $\mathrm{OH}$ bending, respectively, which is characteristic of $\beta$-glycosidic linkages between glucose in the cellulose [37], these two bands also appear in all spectra.

\subsection{X-Ray diffraction}

The cellulose has a well-defined crystalline structure contrary to hemicelluloses and lignin, which are amorphous polymer in nature. The crystalline structure of cellulose is related to the hydrogen bonding interactions and Van der Waals forces between adjacent molecules [38]. The XRD analysis was completed to evaluate the structure and crystallinity of the studied materials at different chemical treatment stages. The XRD patterns of $\mathrm{SCB}, \mathrm{ATB}, \mathrm{BC}, \mathrm{H}-\mathrm{CNC}_{2 \mathrm{M}}, \mathrm{H}-\mathrm{CNC}_{3 \mathrm{M}}$, and $\mathrm{S}-\mathrm{CNC}$ are shown in Figure 3. These patterns are typical of semicrystalline materials with an amorphous broad hump and crystalline peaks. 


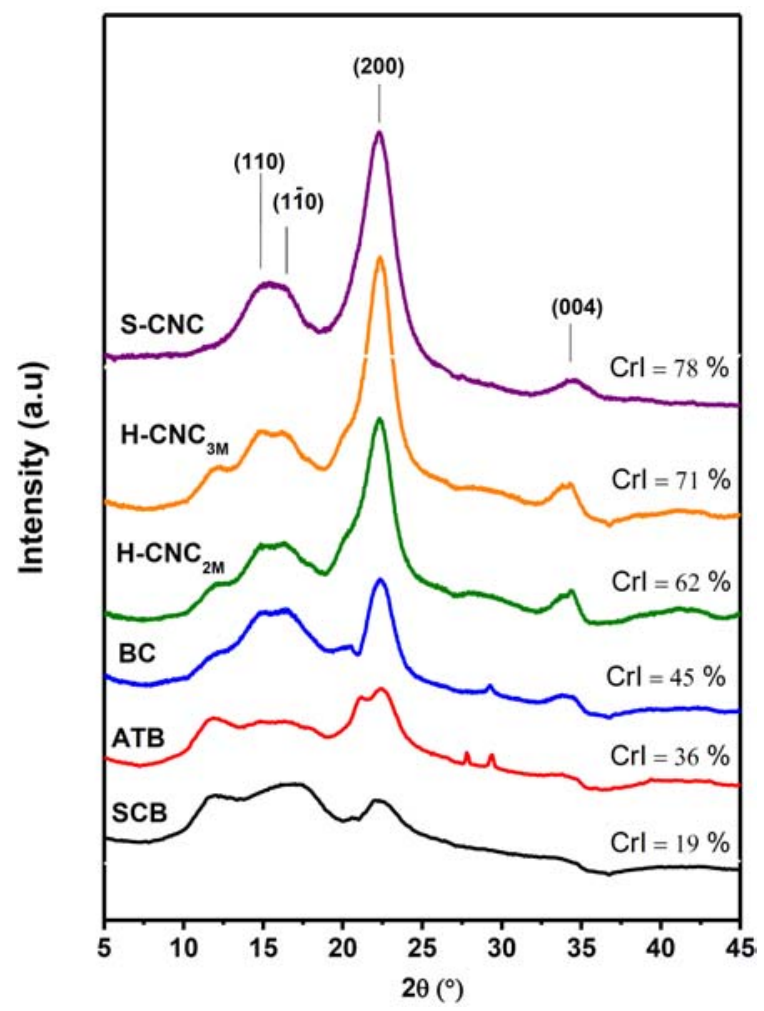

Figure 3. X-ray diffraction patterns of $\mathrm{SCB}, \mathrm{ATB}, \mathrm{BC}, \mathrm{H}-\mathrm{CNC}_{2 \mathrm{M}}$, $\mathrm{H}-\mathrm{CNC}_{3 \mathrm{M}}$, and $\mathrm{S}-\mathrm{CNC}$ samples.

It is noted that in all patterns (Figure 3), there is a predominance of type I cellulose, verified by the presence of peaks at $2 \theta=14.9^{\circ}, 16.8^{\circ}$, $22.6^{\circ}$, and $34.2^{\circ}$ corresponding to the (101), 101-), (002), (004) planes $[33,39]$. The $\mathrm{X}$-ray diffractograms showed that the main intensity peak is located at a $2 \theta$ value of around $22.6^{\circ}$, which is related to the crystalline structure of cellulose I, moreover; this diffraction peak became more intense upon different chemical treatments. This observation could reveal better defined crystalline domains of obtained CNC with well defined orientation along a particular axis, as the non-cellulosic compounds are removed and the amorphous zones of cellulose are dissolved. 
The crystallinity indices (CrI) of SCB, ATB, BC, H-CNC $2 \mathrm{M}, \mathrm{H}-\mathrm{CNC}_{3 \mathrm{M}}$, and S-CNC samples were found to be $19 \%, 36 \%, 45 \%, 62 \%, 71 \%$, and $78 \%$, respectively. These results clearly demonstrate that the crystallinity of the material progressively increases during the chemical extractions. This was ascribed to the progressive removal of amorphous hemicelluloses and lignin by alkaline and bleaching treatment from the raw $\mathrm{SCB}$ to $\mathrm{BC}$ and the removal of amorphous cellulose by acid hydrolysis from $\mathrm{BC}$ to $\mathrm{CNC}\left(\mathrm{H}-\mathrm{CNC}_{2 \mathrm{M}}, \mathrm{H}-\mathrm{CNC}_{3 \mathrm{M}}\right.$, and $\left.\mathrm{S}-\mathrm{CNC}\right)$. The increase in crystallinity after acid treatment was also previously observed [13, 15, 40]. For both kinds of hydrochloric acid hydrolyzed CNC $\left(\mathrm{H}-\mathrm{CNC}_{2 \mathrm{M}}\right.$ and $\left.\mathrm{H}-\mathrm{CNC}_{3 \mathrm{M}}\right)$, it should be noted that the acid concentration has a clear effect on the CNC crystallinity, while the crystallinity increases from $62 \%$ to $71 \%$ when the acid concentration increases from $2 \mathrm{M}$ to $3 \mathrm{M}$. This can be explained by the fact that the amorphous regions of cellulose can be more dissolved when the acid concentration increased, yielding highly crystalline H-CNC. This explanation is further confirmed by the decreasing of $\mathrm{H}$-CNC diameter with increasing of acid concentration, as measured by AFM characterization (see below). Therefore, the amorphous regions are the first to be targeted by the strong acid, followed by regions of increased crystallinity. A controlled hydrolysis can therefore extract regions of a specific crystallinity from a cellulose product. Herein, a concentration of $2 \mathrm{M}$ was insufficient to solubilize the total amorphous regions of cellulose, resulting in CNC with relatively low crystallinity. Then, the concentration of $3 \mathrm{M}$ can solubilize more amorphous regions in regard to that of $2 \mathrm{M}$. The increasing of crystallinity with acid concentration increasing is previously reported [41]. Comparatively, it is found that the $\mathrm{CrI}$ of the S-CNC was higher than that of $\mathrm{H}-\mathrm{CNC}$. The sulphuric acid is more aggressive to pure cellulose than hydrochloric acid, and therefore, it attacks the disordered region is more, yielding highly crystalline S-CNC in regard to $\mathrm{H}-\mathrm{CNC}$. 


\subsection{Thermal analysis}

To investigate the thermal behaviour of the three kinds of CNC ( $\mathrm{H}-\mathrm{CNC}_{2 \mathrm{M}}, \mathrm{H}-\mathrm{CNC}_{3 \mathrm{M}}$, and $\mathrm{S}-\mathrm{CNC}$ ), a thermogravimetric analysis were performed, and the TGA and DTG curves obtained in air atmosphere are illustrated in Figure 4. It is believed that the cellulosic materials degrade at low to moderate temperature [18, 42]. In general, the thermal decomposition of cellulosic materials begins at lower temperature for hemicelluloses followed by an early stage of pyrolysis of lignin and then decomposition of cellulose [43]. Herein, for all samples, an initial weight loss occurs below $100^{\circ} \mathrm{C}$ regardless of their treatment (Figure 4). This degradation is due to the vaporization of water, because of the hydrophilic character of the lignocellulosic fibers or low molecular weight compounds that can be present in these materials [44].

The SCB and ATB fibers, both undergo two-step degradation process. It is noted that the degradation of SCB and ATB fibers follow almost identical mechanism, as demonstrated by DTG curves (Figures 4(a) and 4(b)). The first stage degradation of SCB and ATB fibers occurs at $214-375^{\circ} \mathrm{C}$ and $222-385^{\circ} \mathrm{C}$, respectively. The corresponding TDG curves of these two materials showed a broadening or shoulder peak with maximum at $300^{\circ} \mathrm{C}$, indicating that several specimens have been decomposed in this degradation stage. It has been reported that untreated plant fibers began to degrade around $206^{\circ} \mathrm{C}$ due to low decomposition temperature of hemicelluloses, lignin, and pectin [45]. Herein, the onset degradation temperatures of SCB and ATB fibers is due to the decomposition of non-cellulosic components and mainly lignin and hemicelluloses that present in the SCB and still present in the ATB fibers. In addition, this stage includes also the depolymerisation and dehydratation of cellulose at around $310^{\circ} \mathrm{C}$. The second degradation step (DTG peak above $400-420^{\circ} \mathrm{C}$ ) is attributed to the oxidation and breakdown of the charred residue to lower molecular weight gaseous products [33]. The high charred residue observed in the SCB and ATB fibers is due to the fact that the non-cellulosic could induce higher char formation [33]. 

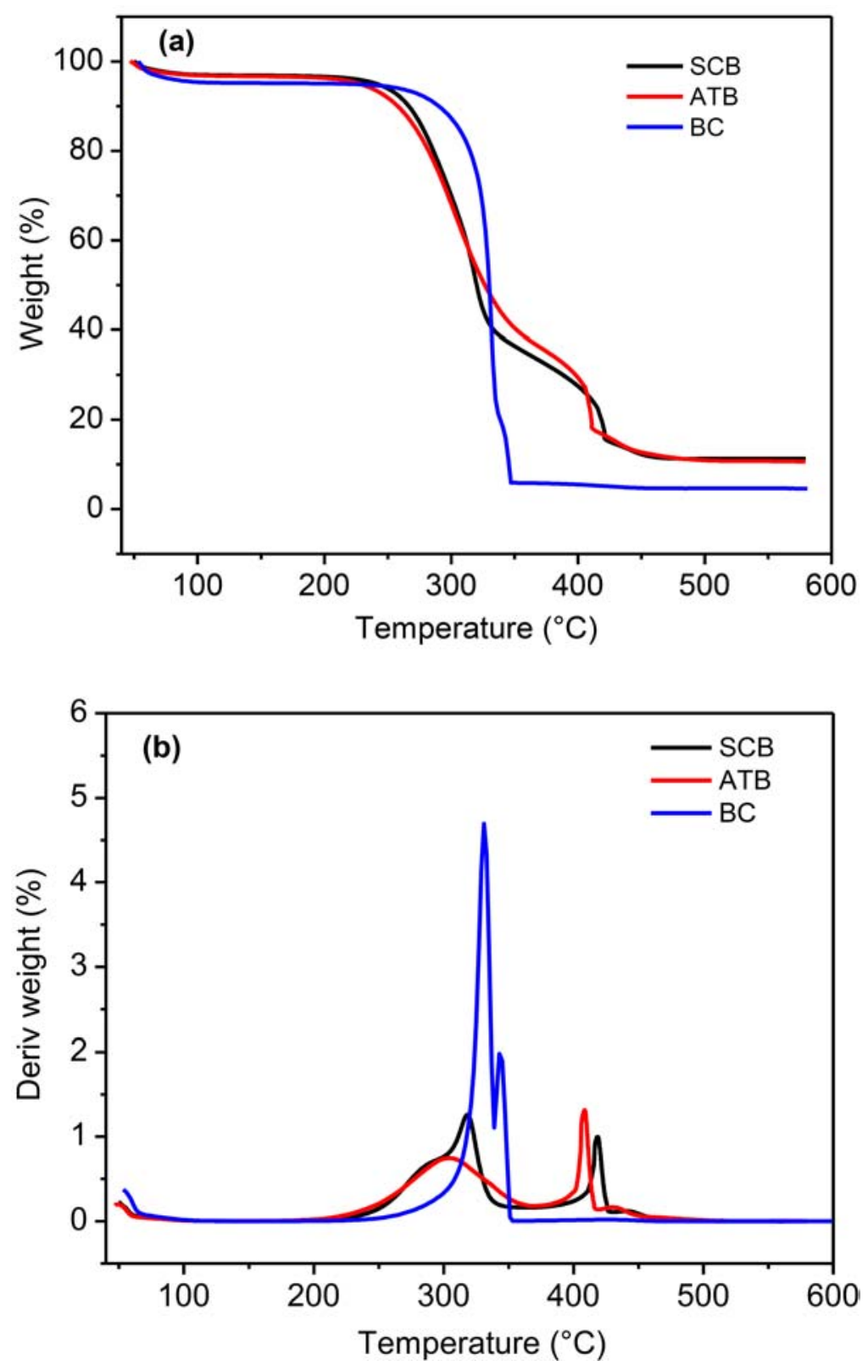

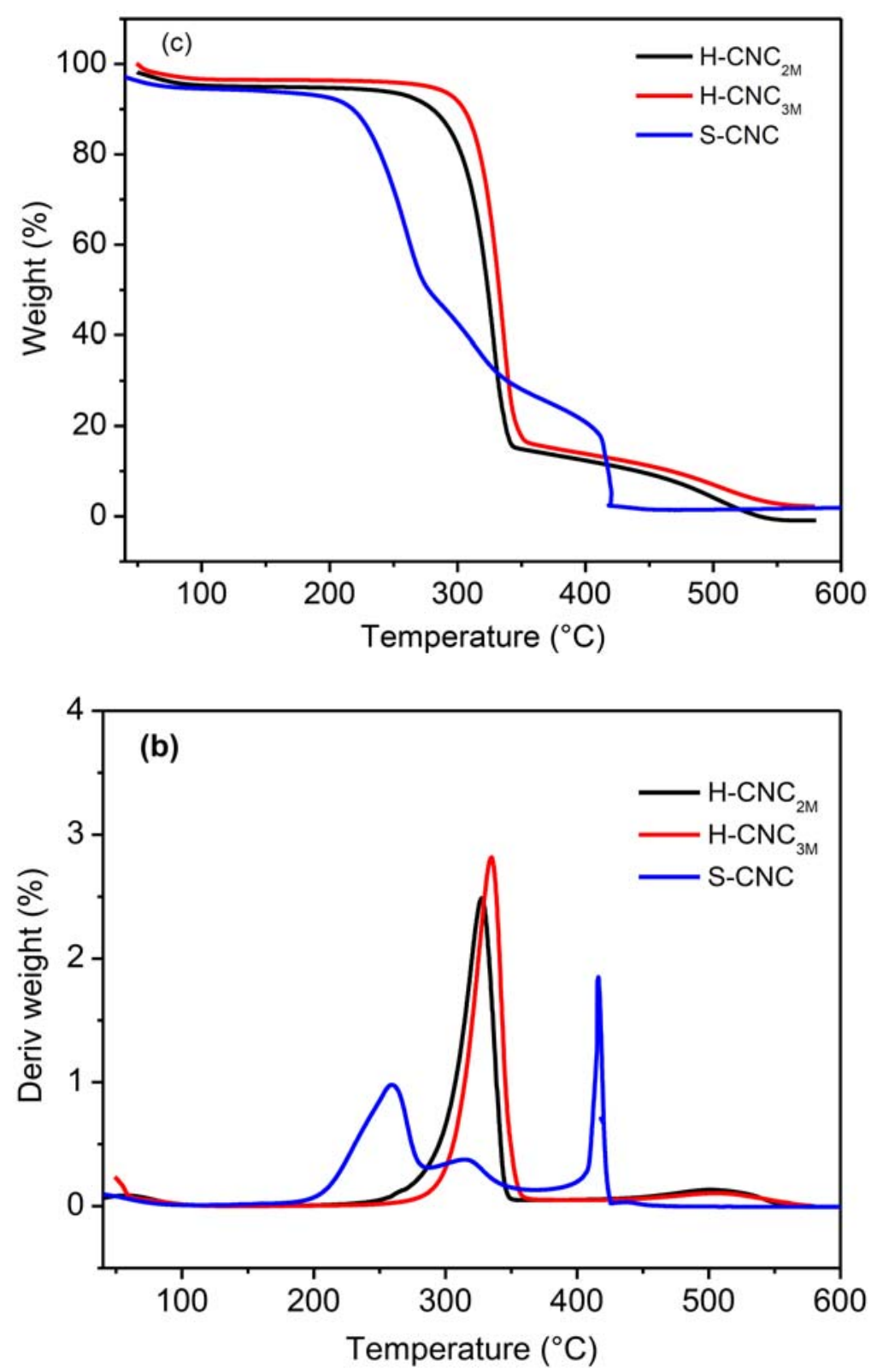

Figure 4. (a, b) TGA and DTG curves of SCB, ATB, and BC samples and (c, d) TGA and DTG curves of H-CNC $2 \mathrm{M}, \mathrm{H}-\mathrm{CNC}_{3 \mathrm{M}}$, and $\mathrm{S}-\mathrm{CNC}$ samples. 
Importantly, it can be seen that the onset thermal decomposition shifts to higher temperature $\left(260^{\circ} \mathrm{C}\right)$ for $\mathrm{BC}$ fibers, and this is due to the process of purification which lead to the elimination of lignin and hemicelluloses residues (Figures 4(a) and 4(b)). Consequently, the lower onset degradation temperature of ATB fibers compared to the BC fibres confirms that the noncellulosic compounds have been partially removed by alkaline treatment. One can be see for BC fibers that the degradation is occurred in one stage degradation process, which corresponds to the depolymerization of pure cellulose $[11,46]$. The TGA analysis showed minimum residual mass for the BC fibers in comparison with SCB and ATB fibers, which probably due to the absence of the charred residue that can be generated from the degradation of lignin and hemicelluloses, however, these molecules have been totally removed by bleaching treatment.

The TGA and DTG curves of $\mathrm{H}-\mathrm{CNC}_{2 \mathrm{M}}, \mathrm{H}-\mathrm{CNC}_{3 \mathrm{M}}$, and S-CNC are shown in Figures 4(c) and 4(d), which allow a quantitative description of the as-isolated CNC's thermal stability. One can be see that the both kinds of hydrochloric acid hydrolyzed CNC have high thermal stability in regard to those obtained by sulphuric acid. The onset degradation temperatures of $\mathrm{H}-\mathrm{CNC}_{2 \mathrm{M}} \quad\left(262^{\circ} \mathrm{C}\right)$ and $\mathrm{H}_{-}-\mathrm{CNC}_{3 \mathrm{M}} \quad\left(275^{\circ} \mathrm{C}\right)$ are approximately $62^{\circ} \mathrm{C}$ and $75^{\circ} \mathrm{C}$ higher than that observed for S-CNC $\left(200^{\circ} \mathrm{C}\right)$. The degradation of $\mathrm{H}-\mathrm{CNC}_{2 \mathrm{M}}$ and $\mathrm{H}-\mathrm{CNC}_{3 \mathrm{M}}$ occurs at one stage with peak rate of degradation is reached at $325^{\circ} \mathrm{C}$ for $\mathrm{H}-\mathrm{CNC}_{2 \mathrm{M}}$ and $334^{\circ} \mathrm{C}$ for $\mathrm{H}-\mathrm{CNC}_{3 \mathrm{M}}$. As suggested for $\mathrm{BC}$ fibers, this degradation is associated to the decomposition of pure cellulose. By comparing the effect of the hydrochloric acid concentration, the onset temperature observed for $\mathrm{H}-\mathrm{CNC}_{3 \mathrm{M}}$ is $14^{\circ} \mathrm{C}$ higher than of $\mathrm{H}-\mathrm{CNC}_{2 \mathrm{M}}$, because the former have high crystallinity that the latter, as measured by XRD characterization (see above). 
On the other hand, S-CNC showed multiple maximums of degradation localized at 261,320 , and $417^{\circ} \mathrm{C}$. The sulphate groups inserted on the surface of S-CNC caused this thermal instability as compared to H-CNC [22, 32, 33]. Consequently, the lack of additional functional groups on the surface of $\mathrm{H}-\mathrm{CNC}$ is the main reason why the $\mathrm{H}$-CNC have a greater thermal stability compared to the S-CNC. As reported previously, the thermal stability is higher when there is little access in the sulphate groups on the surface of sulphuric acid hydrolyzed CNC [47].

\subsection{Aqueous stability of CNC}

Acid hydrolysis carried out under appropriate conditions allows the removal of amorphous domains from the bleached cellulose by cleaving cellulose microfibrils into bundles of CNC. These CNC, which usually range in diameter from 5 to $25 \mathrm{~nm}$ for many cellulosic materials, become more or less individualized by sonication. Figure 5 illustrates aqueous dispersions of both kinds of hydrochloric acid hydrolyzed $\mathrm{CNC}$ ( $\mathrm{H}-\mathrm{CNC}_{2 \mathrm{M}}$ and $\mathrm{H}-\mathrm{CNC}_{3 \mathrm{M}}$ ) and sulphuric acid hydrolyzed CNC (S-CNC) obtained by sonication process after acid hydrolysis. These images are taken one day after the ultrasonication treatment. In general, the dispersibility of CNC depends strongly on their size, surface functionalization, and the ability of the solvent and surface groups to counterbalance the attractive hydrogen-bond interactions exerted by the abundant hydroxyl groups [22]. Herein, the as-obtained $\mathrm{H}-\mathrm{CNC}_{2 \mathrm{M}}$ and $\mathrm{H}-\mathrm{CNC}_{3 \mathrm{M}}$ are stable in water for the chosen short time (one day), and the suspensions had a white gel appearance (Figures 5(a) and 5(b)). As recently demonstrated, the hydrochloric acid hydrolyzed CNC can form a homogeneous stable aqueous dispersion over 10 days [22]. This is due to the hydrophilic nature of CNC which related to the presence of hydroxyl groups in their surface, showing a high affinity for water. However, for a period greater than 10 days, the hydrochloric acid hydrolyzed CNC can start to agglomerate due to the strong hydrogen bonding between their

surface hydroxyl groups [22]. In contrast, it is well know that the 
aqueous dispersion of sulphuric acid hydrolyzed CNC is stable for a long period time, because the presence of negatively charged sulphate groups on their surface causing the strong electrostatic repulsion between nanocrystals. For our S-CNC suspension, the zeta potential has a mean value of $-61.9 \mathrm{mV}$. The suspension of $\mathrm{S}-\mathrm{CNC}$ is considered stable (Figure $5(\mathrm{c})$ ), because the absolute value is higher than $25 \mathrm{mV}$ [32].
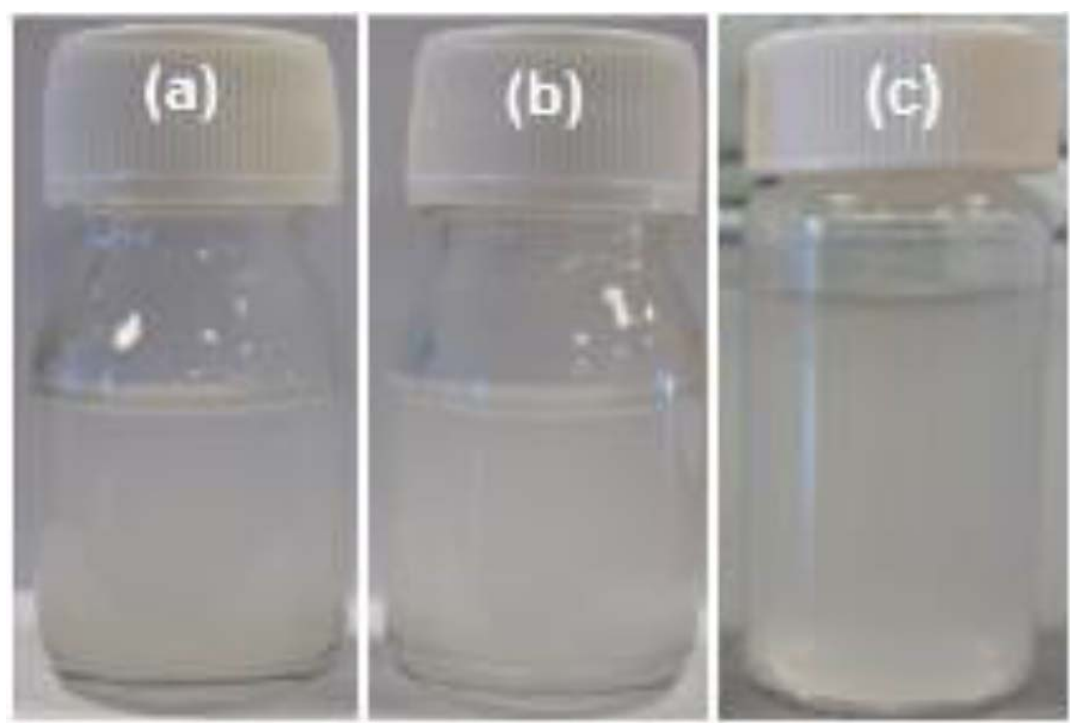

Figure 5. Aqueous suspensions of (a) $\mathrm{H}-\mathrm{CNC}_{2 \mathrm{M}}$, (b) $\mathrm{H}-\mathrm{CNC}_{3 \mathrm{M}}$, and (c) S-CNC samples.

\subsection{AFM analysis of CNC}

The morphology and dimension of as-obtained $\mathrm{H}-\mathrm{CNC}_{2 \mathrm{M}}, \mathrm{H}-\mathrm{CNC}_{3 \mathrm{M}}$, and S-CNC are characterized by AFM analysis. It has been reported that the AFM microscopy is an effective technique to characterize the morphology and size of CNC [17]. Figure 6 illustrates AFM images for $\mathrm{H}-\mathrm{CNC}_{2 \mathrm{M}}, \mathrm{H}-\mathrm{CNC}_{3 \mathrm{M}}$, and $\mathrm{S}-\mathrm{CNC}$ and the width and length of all $\mathrm{CNC}$ were determined from AFM height images. From these results, it has been confirmed that all $\mathrm{CNC}$ have needle-like nanoparticles, thus confirming that their extraction was successful from treated SCB. 

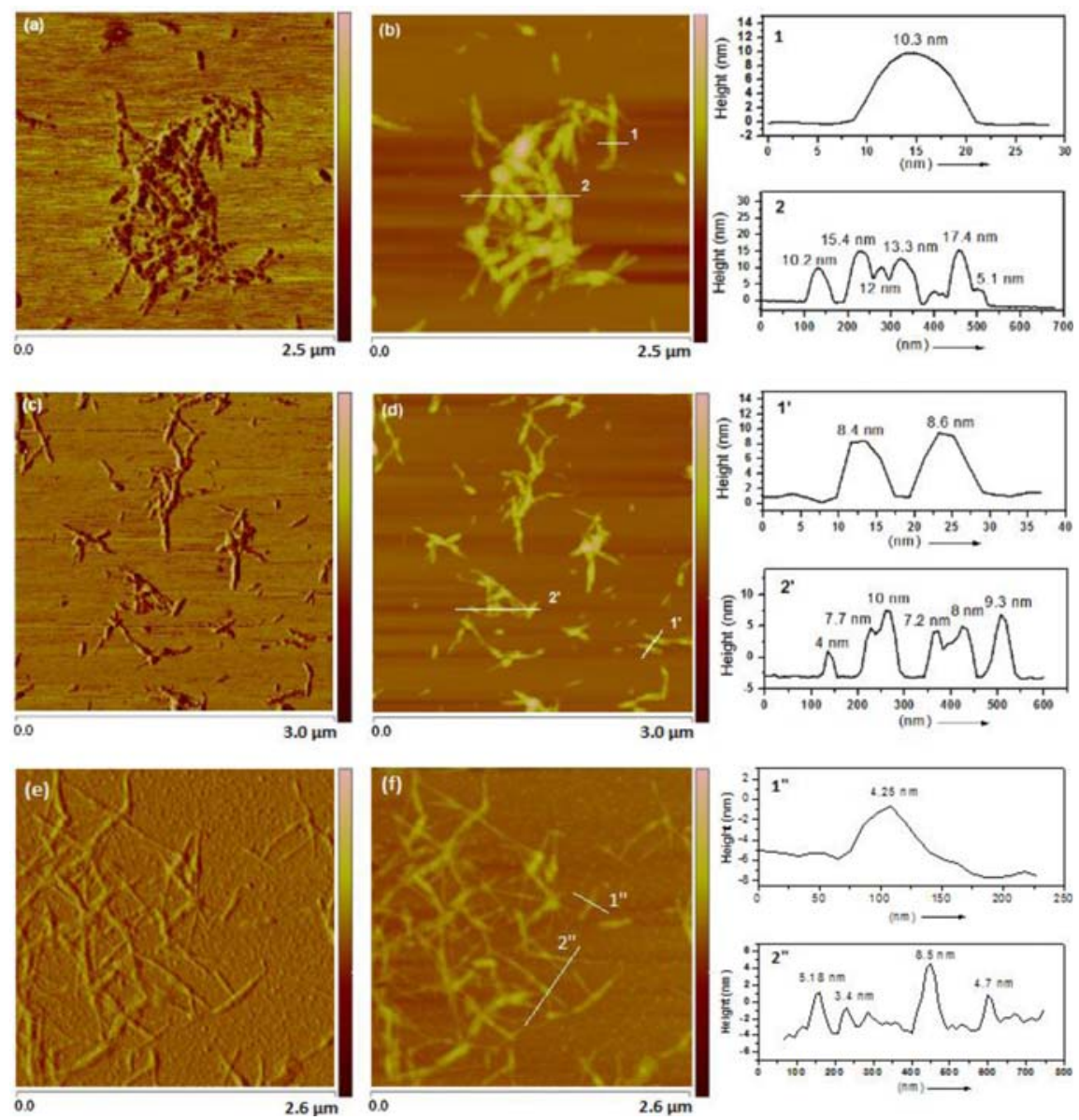

Figure 6. Phase and height images of tapping mode $\mathrm{AFM}$ of $(\mathrm{a}, \mathrm{b})$ $\mathrm{H}-\mathrm{CNC}_{2 \mathrm{M}}$, (c, d) $\mathrm{H}-\mathrm{CNC}_{3 \mathrm{M}}$, and (e, f) S-CNC. For the three kinds of $\mathrm{CNC}$, two height profiles taken along the indicated circle are presented.

All images showed individual $\mathrm{CNC}$ and some aggregates through transverse height profiles. The appearance of laterally aggregated elementary crystallites in AFM images is expected due to the high specific area and strong hydrogen bonds established between the CNC. Additionally, AFM images show that all extracted $\mathrm{CNC}\left(\mathrm{CNC}_{2 \mathrm{M}}\right.$, $\mathrm{CNC}_{3 \mathrm{M}}$, and $\left.\mathrm{S}-\mathrm{CNC}\right)$ are irregular in diameter and length. In general, the exact dimensions of the $\mathrm{CNC}$ depend on the nature of the original 
raw material and hydrolysis conditions or pretreatments [27]. Herein, the dimensions of the three kinds of CNC were approximately calculated by the high profiles taken vertically along the some CNC (Figure 6). For both kinds of hydrochloric acid hydrolyzed CNC, it is clear that the diameter is in nanometric scale ranging from 9 to $20 \mathrm{~nm}$ for $\mathrm{CNC}_{2 \mathrm{M}}$ and 4 to $10 \mathrm{~nm}$ for $\mathrm{CNC}_{3 \mathrm{M}}$. The diameter of both $\mathrm{H}-\mathrm{CNC}$ was calculated in the range of $100-400 \mathrm{~nm}$. Comparatively, it is clear that the $\mathrm{CNC}_{3 \mathrm{M}}$ have smaller diameter in regard to $\mathrm{CNC}_{2 \mathrm{M}}$. This variation in diameter is due to the hydrolysis conditions of both $\mathrm{CNC}$, which in our case the variation of acid concentration, while the increase in acid concentration resulted in a slight smaller diameter for $\mathrm{CNC}_{3 \mathrm{M}}$ compared to the $\mathrm{CNC}_{2 \mathrm{M}}$. This augmentation led to the high solubility of the amorphous region, as confirmed and discussed by the XRD measurements (Figure 3), showing that the crystallinity index of the hydrolyzed CNC increased by increasing acid concentration. On the other hand, the sulphuric acid hydrolyzed CNC (S-CNC) showed a diameter and a length in the range of 3-9nm and 150-500nm, respectively. The results obtained for S-CNC are in good agreement, in term of geometry and diameter scale, with those observed by Teixeira et al. for CNC obtained from SCB by $6 \mathrm{M}$ sulphuric acid hydrolysis [11] and also for CNC obtained from others lignocellulosic materials [48-51]. From these results, we concluded that the nanocrystals' dimensions are influenced by the hydrolysis conditions, such as acid type and concentration. Herein, the use of SCB as raw material is a route if $\mathrm{CNC}$ with specific dimensions are required by industrial applications, such as polymer nanocomposite development.

\section{Conclusion}

In the aim of valorizing by-products of the Moroccan sugar mills, we have shown in the present work that the pure cellulose and cellulose nanocrystals can be successfully produced from sugarcane bagasse by chemical methods. It was found that the alkaline and bleaching treatments removed the non-cellulosic constituents resulting in fibers

with pure cellulose, hence suitable for extracting of nanocrystals. Three 
kinds of cellulose nanocrystals have been isolated by acid hydrolysis using sulphuric acid at a concentration of $9 \mathrm{M}$ for $30 \mathrm{~min}$ and hydrochloric acid at two concentrations of $2 \mathrm{M}$ and $3 \mathrm{M}$ for $120 \mathrm{~min}$. Characterization techniques through FTIR, XRD, ATG, and AFM showed that the CNC have been successfully isolated with high crystallinity, nanometric scale and good thermal stability. It was found that the properties of asextracted CNC are influenced by the extraction conditions. The FTIR analysis confirmed that pure cellulose has been observed for bleached cellulose fibers and CNC, which is achieved by removal of lignin, hemicellulose and all other non-cellulosic compounds. The XRD results revealed that the crystallinity index of the CNC was strongly depend of the acid attack degree and the acid concentration and it was found in the range of $62-78 \%$ and the all obtained $\mathrm{CNC}$ have the typical cellulose I structure. The TGA analysis showed that the hydrochloric acid hydrolyzed CNC exhibited a good thermal stability in regard to that obtained for sulphuric acid hydrolyzed CNC, which are frequently employed, but suffer from limited thermal stability. The AFM measurements showed that the three kinds of CNC exhibited needle-like nanoparticles with a diameter range of $3-25 \mathrm{~nm}$ and a length of 100-500 depending on the hydrolysis conditions. From our results, it can be concluded that the CNC obtained from Moroccan sugarcane bagasse have excellent physico-chemical properties. Therefore, they can be potentially used in various applications. Through this study, we have demonstrated a possible strategy to give an added value to Moroccan sugarcane bagasse.

\section{Acknowledgement}

The financial assistance of the OCP Group (Office Chérifien des Phosphates in the Moroccan Kingdom) towards this research is hereby acknowledged. The authors are also grateful to COSUMAR Company in Morocco for providing us the raw sugarcane bagasse. We equally thank all administrative and technical support teams of the 'Université Mohammed VI Polytechnique'. 


\section{References}

[1] C. O. Tuck, E. Pérez, I. T. Horváth, R. A. Sheldon and M. Poliakoff, Valorization of biomass: Deriving more value from waste, Science 337 (2012), 695.

[2] E. A. Martínez, S. S. Silva, J. B. A. Silva, A. I. N. Solenzal and M. G. A. Felipe, The influence of $\mathrm{pH}$ and dilution rate on continuous production of xylitol from sugarcane bagasse hemicellulosic hydrolysate by C. Guilliermondii, Process Biochem. 38 (2003), 1677.

[3] J. M. Hernández-Salas, M. S. Villa-Ramírez, J. S. Veloz-Rendón, K. N. RiveraHernández, R. A. González-César and M. A. Plascencia-Espinosa, Comparative hydrolysis and fermentation of sugarcane and agave bagasse, Bioresour. Technol. 100 (2009), 1238.

[4] A. K. Chandel, R. K. Kapoor, A. Singh and R. C. Kuhad, Detoxification of sugarcane bagasse hydrolysate improves ethanol production by candida shehatae NCIM 3501, Bioresour. Technol. 98 (2007), 1947.

[5] K. K. Cheng, B. Y. Cai, J. A. Zhang, H. Z. Ling, Y. J. Zhou, J. P. Ge and J. M. Xu, Sugarcane bagasse hemicellulose hydrolysate for ethanol production by acid recovery process, Biochem. Eng. J. 38 (2008), 105.

[6] D. Restuti and A. Michaelowa, The economic potential of bagasse cogeneration as CDM projects in Indonesia, Energ. Policy 35 (2007), 3952.

[7] J. A. Quintero, M. I. Montoya, O. J. Sánchez, O. H. Giraldo and C. A. Cardona, Fuel ethanol production from sugarcane and corn: Comparative analysis for a Colombian case, Energy 33 (2008), 385.

[8] X. F. Sun, F. Xu, R. C. Sun, P. Fowler and M. S. Baird, Characteristics of degraded cellulose obtained from steam-exploded wheat straw, Carbohydr. Res. 340 (2005), 97.

[9] A. Moubarik and N. Grimi Boussetta, Structural and thermal characterization of Moroccan sugarcane bagasse cellulose fibers and their applications as a reinforcing agent in low density polyethylene, Compos. Part B Eng. 52 (2013), 233.

[10] J. Li, X. Wei, Q. Wang, J. Chen, G. Chang, L. Kong, J. Su and Y. Liu, Homogeneous isolation of nanocellulose from sugarcane bagasse by high pressure homogenization, Carbohydr. Polym. 90 (2012), 1609.

[11] E. D. M. Teixeira, T. J. Bondancia, K. B. R. Teodoro, A. C. Corrêa, J. M. Marconcini and L. H. C. Mattoso, Sugarcane bagasse whiskers: Extraction and characterizations, Ind. Crop. Prod. 33 (2011), 63.

[12] R. G. P. Viera, G. R. Filho, R. M. N. D. Assunção, C. D. S. Meireles, J. G. Vieira and G. S. D. Oliveira, Synthesis and characterization of methylcellulose from sugarcane bagasse cellulose, Carbohydr. Polym. 67 (2007), 182.

[13] A. Mandal and D. Chakrabarty, Isolation of nanocellulose from waste sugarcane bagasse (SCB) and its characterization, Carbohydr. Polym. 86 (2011), 1291. 
[14] J. Bras, M. L. Hassan, C. Bruzesse, E. A. Hassan, N. A. El Wakil and A. Dufresne, Mechanical, barrier, and biodegradability properties of bagasse cellulose whiskers reinforced natural rubber nanocomposites, Ind. Crop. Prod. 32 (2010), 627.

[15] M. A. S. Azizi-Samir, F. Alloin, J. Y. Sanchez and A. Dufresne, Cellulose nanocrystals reinforced poly (oxyethylene), Polymers 45 (2004), 4149.

[16] E. Fortunati, D. Puglia, M. Monti, L. Peponi, C. Santulli, J. M. Kenny and L. Torre, Extraction of cellulose nanocrystals from phormium tenax fibres, J. Polym. Environ. 21 (2013), 319.

[17] P. Lu and Y. L. Hsieh, Preparation and characterization of cellulose nanocrystals from rice straw, Carbohydr. Poly. 87 (2012), 564.

[18] N. Rehman, M. I. G. de Miranda, S. M. L. Rosa, D. M. Pimentel, S. M. B. Nachtigall and C. I. D. Bica, Cellulose and nanocellulose from maize mtraw: An insight on the crystal properties, J. Polym. Environ. 22 (2014), 252.

[19] J. Yang, C. R. Han, J. F. Duan, F. Xu and R. C. Sun, Mechanical and viscoelastic properties of cellulose nanocrystals reinforced poly (ethylene glycol) nanocomposite hydrogels, ACS Appl. Mater. Interfaces 5 (2013), 3199.

[20] H. Yuan, Y. Nishiyama, M. Wada and S. Kuga, Surface acylation of cellulose whiskers by drying aqueous emulsion, Biomacromolecules 7 (2006), 696.

[21] R. F. Nickerson and J. A. Habrle, Cellulose intercrystalline structure study by hydrolytic methods, Ind. Eng. Chem. 39 (1947), 1507.

[22] S. C. Espinosa, T. Kuhnt, E. J. Foster and C. Weder, Isolation of thermally stable cellulose nanocrystals by phosphoric acid hydrolysis, Biomacromolecules 14 (2013), 1223.

[23] M. F. Rosa, E. S. Medeiros, J. A. Malmonge, K. S. Gregorski, D. F. Wood, L. H. C. Mattoso, G. Glenn, W. J. Orts and S. Imam, Cellulose nanowhiskers from coconut husk fibers: Effect of preparation conditions on their thermal and morphological behavior, Carbohydr. Polym. 81 (2010), 83.

[24] H. Sadeghifar, I. Filpponen, S. P. Clarke, D. F. Brougham and D. S. Argyropoulos, Production of cellulose nanocrystals using hydrobromic acid and click reactions on their surface, J. Mater. Sci. 46 (2011), 7344.

[25] J. Araki, M. Wada, S. Kuga and T. Okano, Birefringent glassy phase of a cellulose microcrystal suspension, Langmuir. 16 (2000), 2413.

[26] R. Zuluaga, JL. Putaux, J. Cruz, J. Vélez, I. Mondragon and P. Ganan, Cellulose microfibrils from banana rachis: Effect of alkaline treatments on structural and morphological features, Carbohydr. Polym. 76 (2009), 51.

[27] S. J. Eichhorn, A. Dufresne, M. Aranguren, N. E. Marcovich, J. R. Capadona and S. J. Rowan et al., Review: Current international research into cellulose nanofibres and nanocomposites, J. Mater. Sci. 45 (2010), 1. 
[28] Y. Habibi, I. Hoeger, S. S. Kelley and O. J. Rojas, Development of LangmuirSchaeffer cellulose nanocrystal monolayers and their interfacial behaviors, Langmuir. 26 (2010), 990.

[29] L. Segal, J. J. Creely, A. E. Martin and C. M. Conrad, The empirical method for estimating the degree of cristallinity of native cellulose using the X-ray diffractometer, Tex. Res. J. 29 (1959), 786.

[30] R. M. Dos Santos, W. P. F. Neto, H. A. Silvério, D. F. Martins, N. O. Dantas and D. Pasquini, Cellulose nanocrystals from pineapple leaf, a new approach for the reuse of this agro-waste, Ind. Crop. Prod. 50 (2013), 707.

[31] B. Deepa, E. Abraham, B. M. Cherian, A. Bismarck, J. J. Blaker and L. A. Pothan, Structure, morphology and thermal characteristics of banana nanofibers obtained by steam explosion, Bioresour. Technol. 102 (2011), 1988.

[32] J. P. S. Morais, M. D. F. Rosa, M. D. M. D. S. Filho, L. D. Nascimento, D. M. D. Nascimento and A. R. Cassales, Extraction and characterization of nanocellulose structures from raw cotton linter, Carbohydr. Polym. 91 (2013), 229.

[33] W. P. F. Flauzino-Neto, H. A. Silvério, N. O. Dantas and D. Pasquini, Extraction and characterization of cellulose nanocrystals from agro-industrial residue-Soy hulls, Ind. Crop. Prod. 42 (2013), 480.

[34] A. Barakat, C. Gaillard, J. P. Steyer and H. Carrere, Anaerobic biodegradation of cellulose xylan-lignin nanocomposites as model assemblies of lignocellulosic biomass, Waste Biomass Valor. 5 (2014), 293.

[35] P. Lu and YL. Hsieh, Preparation and properties of cellulose nanocrystals: Rods, spheres, and network, Carbohydr. Polym. 5 (2010), 329.

[36] F. Jiang, A. R. Esker and M. Roman, Acid-catalyzed and solvolytic desulfation of $\mathrm{H}_{2} \mathrm{SO}_{4}$-hydrolyzed cellulose nanocrystals, Langmuir. 26 (2010), 17919.

[37] C. F. Liu, J. L. Ren, F. Xu, J. J. Liu, J. X. Sun and R. C. Sun, Isolation and characterization of cellulose obtained from ultrasonic irradiated sugarcane bagasse, J. Agri. Food Chem. 54 (2006), 5742.

[38] Y. H. P. Zhang and L. R. Lynd, Toward an aggregated understanding of enzymatic hydrolysis of cellulose: Non complexed cellulase systems, Biotechnol. Bioeng. 88 (2004), 797.

[39] S. Borysiak and J. Garbarczyk, Applying the WAXS method to estimate the supermolecular structure of cellulose fibres after mercerization, Fibers Text East Eur. 11 (2003), 104.

[40] H. A. Silvério, W. P. Flauzino-Neto, N. O. Dantas and D. Pasquini, Extraction and characterization of cellulose nanocrystals from corncob for application as reinforcing agent in nanocomposites, Ind. Crop. Prod. 44 (2013), 427.

[41] A. M. A. Nada, M. Y. El-Kady, E. S. A. El-Sayed and F. M. Amine, Preparation and characterization of microcrystalline cellulose (MCC), Bioresources 4 (2009), 1359. 
[42] M. Hajaligol, B. Waymack and D. Kellogg, Low temperature formation of aromatic hydrocarbon from pyrolysis of cellulosic materials, Fuel 80 (2001), 1799.

[43] H. L. Lee, G. C. Chen and R. M. Rowell, Thermal properties of wood reacted with a phosphorus pentoxide-amine system, J. Appl. Polym. Sci. 91 (2004), 2465.

[44] N. Johar, I. Ahmad and A. Dufresne, Extraction, preparation and characterization of cellulose fibres and nanocrystals from rice husk, Ind. Crop. Prod. 37 (2012), 93.

[45] W. H. Chen, Y. J. Tu and H. K. Sheen, Disruption of sugarcane bagasse lignocellulosic structure by means of dilute sulfuric acid pretreatment with microwave-assisted heating, Appl. Energ. 88 (2011), 2726.

[46] A. Sonia and K. P. Dasan, Chemical, morphology and thermal evaluation of cellulose microfibers obtained from Hibiscus sabdariffa, Carbohydr Polym. 92 (2013), 668.

[47] N. Wang, E. Ding and R. Cheng, Thermal degradation behaviors of spherical cellulose nanocrystals with sulfate groups, Polymer 48 (2007), 3486.

[48] S. Elazzouzi-Hafraoui, Y. Nishiyama, L. Heux, F. Dubreuil and C. Rochas, The shape and size distribution of crystalline nanoparticles prepared by acid hydrolysis of native cellulose, Biomacromolecules 9 (2008), 57.

[49] M. A. S. Azizi-Samir, F. Alloin and A. Dufresne, Review of recent research into cellulosic whiskers, their properties and their application in nanocomposite field, Biomacromolecules 6 (2005), 612.

[50] J. Araki, M. Wada, S. Kuga and T. Okano, Flow properties of microcrystalline cellulose suspension prepared by acid treatment of native cellulose, Colloids Surf. A Physicochem. Eng. Asp. 142 (1998), 75.

[51] X. Dong, J. F. Revol and D. Gray, Effect of microcrystallite preparation conditions on the formation of colloid crystals of cellulose, Cellulose 5 (1998), 19. 